\title{
2044. Dynamics analysis for the clamping mechanisms of a rotary inchworm piezoelectric motor
}

\author{
Yongfei Gu${ }^{1}$, Jichun Xing² \\ ${ }^{1,2}$ School of Mechanical Engineering, Yanshan University, Qinhuangdao, China \\ ${ }^{1}$ Education Ministry Key Laboratory of Advanced Forging and Stamping Technology and Science, \\ Yanshan University, Qinhuangdao, China \\ ${ }^{2}$ Corresponding author

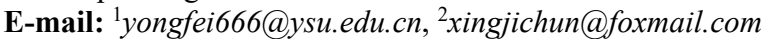

Received 3 November 2015; received in revised form 23 January 2016; accepted 2 February 2016 DOI http://dx.doi.org/10.21595/jve.2016.16005

\begin{abstract}
In this study, a rotary inchworm piezoelectric motor is proposed. The operating principle of the proposed motor and its clamping mechanisms are discussed. Moreover, we also establish the dynamic equation of the clamping mechanisms. From the established equations, the natural frequencies and modal functions are obtained. The sensitivities of the configuration parameter to natural frequencies of the clamping mechanisms are investigated. Finally, the tests on the relative errors of the natural frequencies between the theoretical and experimented results are performed to verity the correctness of the theoretical analysis.
\end{abstract}

Keywords: inchworm principal, piezoelectric motor, clamping mechanisms, free vibration.

\section{Nomenclature}

E Elasticity modulus of the clamping mechanisms material

$b \quad$ Width of the flexible hinge

$t \quad$ Thickness of the flexible hinge recess

$R \quad$ Notch radius of the flexible hinge

$x_{i} \quad$ Length coordinate of the beam

$y_{i} \quad$ Transverse coordinate of the beam

$u_{i}\left(x_{i}, t\right) \quad$ Longitudinal displacement of the beam

$\rho \quad$ Material density of the clamping mechanisms

$\varphi_{u i}\left(x_{i}\right) \quad$ Mode function of the longitudinal vibration of beams

$q(t) \quad$ Time variant function

$D_{1 i}, D_{2 i} \quad$ Constants of $\varphi_{u i}$

$\omega_{i} \quad$ Natural frequency

$w_{i}\left(x_{i}, t\right) \quad$ Transverse displacement of the beam

$\kappa \quad$ Section factor

G Shear modulus

$I_{i} \quad$ Second moment of the area of beam

$S_{i} \quad$ Section area of the beam

$t \quad$ Time

$\varphi_{w i}\left(x_{i}\right) \quad$ Mode function of the bending vibration of beams

$a \quad$ Abscissa of flexible hinge position

$C_{i} \quad$ Constants of $\varphi_{w 1}$

$B_{i} \quad$ Constants of $\varphi_{w 2}$

$\zeta_{B(C)} \quad$ Rotation angles of point $\mathrm{B}(\mathrm{C})$

$M_{B(C)} \quad$ Bending moment of point $\mathrm{B}(\mathrm{C})$

$F_{B(C)} \quad$ Shearing force of point $\mathrm{B}(\mathrm{C})$

$N_{B(C)} \quad$ Pulling force of point $\mathrm{B}(\mathrm{C})$

$l_{1} \quad$ Length of the LAB

$l_{2} \quad$ Length of the LFB

$\theta \quad$ Angle between the LFB and the horizontal

$h \quad$ Thickness of the beam

$h_{1} \quad$ Thickness of the LAB

$h_{2} \quad$ Thickness of the LFB 


\section{Introduction}

Piezoelectric motors, which can be classified by their operation frequency, are used for micro/macro positioning in the longitudinal and rotary directions [1]. Quasi-static motors, such as inchworm piezoelectric motors and inertial impact motors, were operated at low frequency with a stable response. Ultrasonic motors were operated at a high frequency, in which traveling wave ultrasonic motors have been carried out the topic of a large number of studies on and achieved prominent results $[2,3]$. The most common type of rotary piezoelectric motors is the traveling wave ultrasonic motor. Its rotational speed is high but the positioning accuracy and resolution under high-precision positioning is low [4]. By contrast, inchworm piezoelectric motors feature a large stroke with high accuracy and resolution. This type of motors can also overcome the output torque shortage of inertial impact piezoelectric motors [5].

As an important type of piezoelectric motors, inchworm piezoelectric motors have been successfully applied in precision driving [6]. However, most existing inchworm piezoelectric motors are used for positioning in the longitudinal direction. The principle of inchworm motion is rarely applied in precision rotational driving field. Therefore, the application of inchworm piezoelectric motors in precision control are limited [7].

The present study proposes a novel type of rotary inchworm piezoelectric precision motor. A dynamic model of clamping mechanisms (CMs) is also put forward. The natural frequencies and modal functions are obtained. And the effects of the principal factors that influence the natural frequencies are investigated. A prototype motor is finally developed. The experimental results of the natural frequency of the clamping mechanisms are consistent with the theoretical analysis.

\section{Motor description}

The motor consists of a stator and a rotor (see Fig. 1). The stator consists of two CMs, two driving mechanisms (DMs), and a swinger base (SB). The two CMs are symmetrically mounted on an upper SB to grip the rotor alternately. The two DMs are symmetrically mounted on a lower $\mathrm{SB}$ to create an angular displacement. A square wave voltage signal at a special frequency is input to four piezo-stacks to make the motor move (see Fig. 2 for the sequence of voltage signals in a cycle). The operating principle of the motor is shown in Fig. 2.

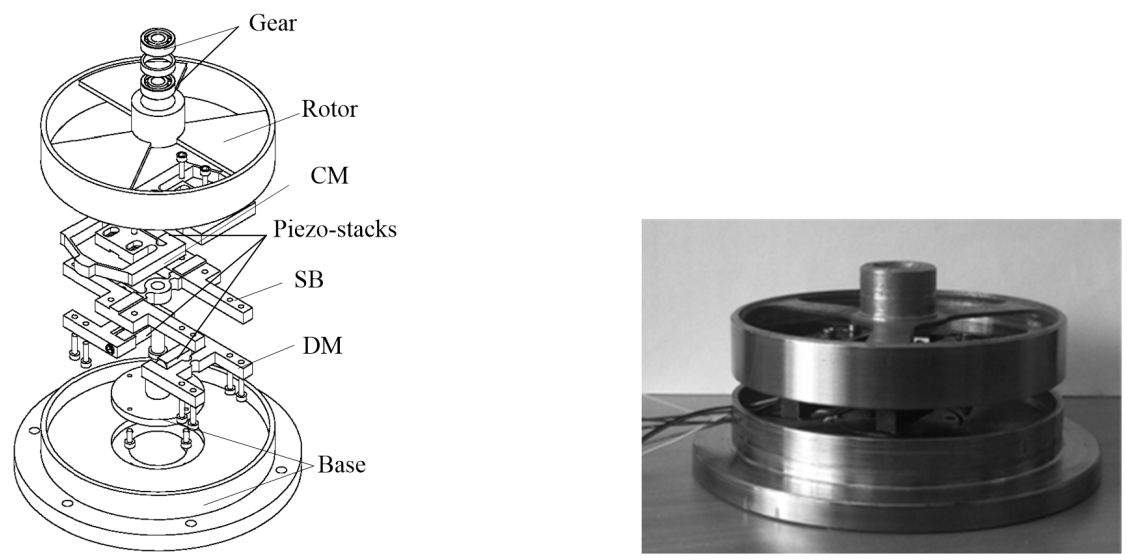

Fig. 1. Rotary inchworm piezoelectric motor

The working procedure of the motor is as follows:

1. The contact shoe of $\mathrm{CM}(\mathrm{A})$ is extended and clamped onto the rotor.

2. As $\mathrm{DM}(\mathrm{A})$ is bent by piezo-stack(B), the arm of SB rotates at an angle $\theta$.

3. The contact shoe of $\mathrm{CM}(\mathrm{A})$ contracts and releases the rotor, while the contact shoe of $\mathrm{CM}(\mathrm{B})$ is extended and clamped onto the rotor. The rotor remains stationary during the process. 
4. The arm of SB is restored to the original state, and the rotor is again rotated at an angle $\theta$.

5. As the $\mathrm{DM}(\mathrm{B})$ is bent by piezo-stack (D), the arm of SB is rotated at an angle $\theta$ again. The contact shoe of $\mathrm{CM}(\mathrm{B})$ contracts and releases the rotor and the arm of SB is restored to its original state. The steps 1 to 5 are repeated to achieve continuous working of the motor

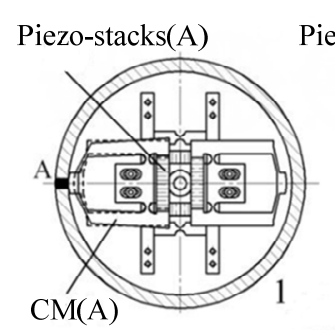

$\mathrm{CM}(\mathrm{A})$

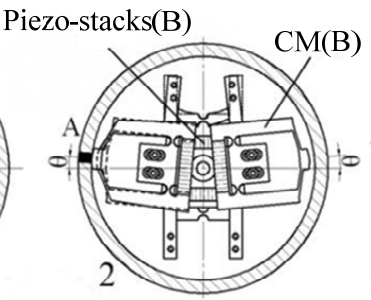

Fig. 2. Driving principal schematic
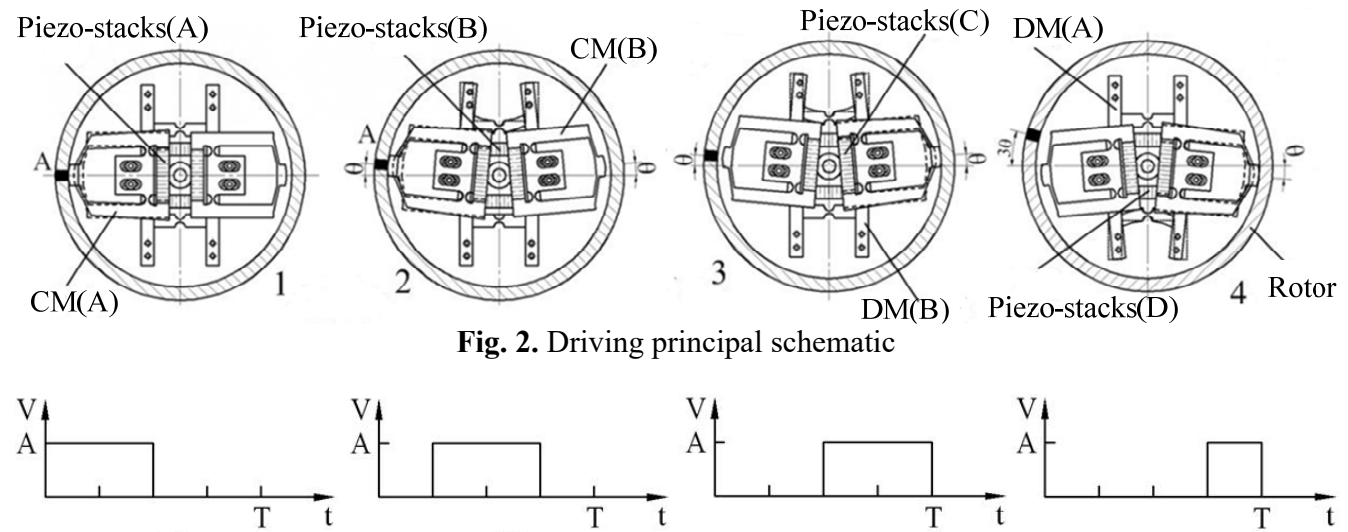

Fig. 3. Driving signal

\section{Dynamics model of the clamping mechanisms}

Each CM which is driven by the piezo-stack integrates lever and triangle amplification to achieve contact shoe displacement amplification. The configuration of CMs is shown in Fig. 4. When the motor is operated, the deformation of the CMs mainly occurred in the lever amplification beam (LAB) and the long flexibility beam (LFB). The symmetric vibration modes of the CMs are considered, because of their symmetric structure. The dynamic of half a CM is analyzed. The simplified dynamic model is based on the following assumptions:

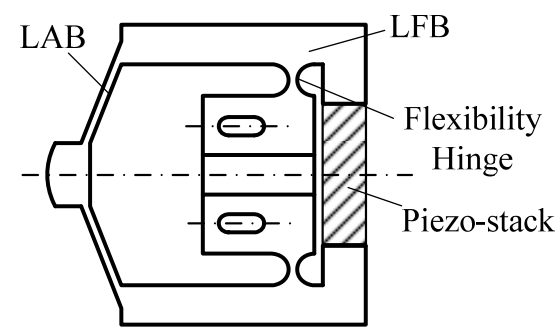

Fig. 4. Displacement amplification principle

1) The ratio of $L A B$ thickness to its length is more than 0.2 . Given the impact of cross-section rotational inertia, the LAB is a Timoshenko beam.

2) The boundary condition on the right end of the LAB is considered as lumped mass $m$.

3) The bending deformation of flexible hinge is considered, while tensile deformation is ignored. Hence, the flexible hinge is considered both as a hinge and as a coil spring. The rotational stiffness equation for a straight round flexible hinge, which is deduced by Yingfei $\mathrm{Wu}$ and Zhaoying Zhou [8], is as follows:

$$
\begin{aligned}
& K=\frac{E b R^{2}}{12 f_{2}} \\
& f_{2}=\frac{2 s^{3}\left(6 s^{2}+4 s+1\right)}{(2 s+1)(4 s+1)^{2}}+\frac{12 s^{4}(2 s+1)}{(4 s+1)^{\frac{5}{2}}} \arctan \sqrt{4 s+1},
\end{aligned}
$$


$s=\frac{R}{t}$

where $E$ is the modulus of elasticity of the material for the CMs, $b$ is the width of the flexible hinge, $t$ is the thickness of the recess of flexible hinge, and $R$ is the notch radius of the flexible hinge.

4) The boundary condition on the right end of the LAB is considered as a fixed end.

5) Considering the bending and longitudinal vibration deformations of the LAB and LFB, the dynamic model of the CMs is simplified into two uniform beams. Fig. 5 describes the two uniform beams, where the $x$-axis is along the $x_{i}(i=1,2)$, the $y$-axis is along the $y_{i}$, and the origin is $\mathrm{B}(\mathrm{C})$.
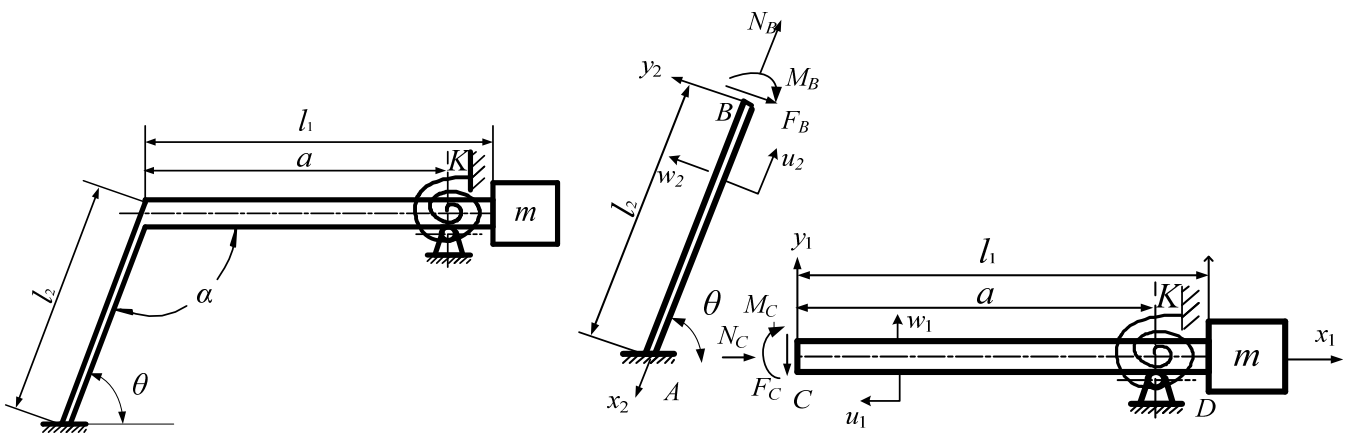

Fig. 5. Dynamic model of the CMs

\subsection{The longitudinal vibration of beams}

Suppose the cross section of the beam in the model remains a plane in the vibration process. The dynamic equation of the beam in the longitudinal vibration is as follows:

$\frac{\partial^{2} u_{i}\left(x_{i}, t\right)}{\partial t^{2}}=\frac{E}{\rho} \frac{\partial^{2} u_{i}\left(x_{i}, t\right)}{\partial x_{i}^{2}}$

where $u_{i}\left(x_{i}, t\right)$ is the displacement of the beam longitudinal direction, $x_{i}$ is the length coordinate of the beam, $t$ is thetime, $\rho$ is the material density of the CMs, and $E$ is the elasticity modulus of the CMs material.

Let $u_{i}\left(x_{i}, t\right)=\varphi_{u i}\left(x_{i}\right) q(t)$. Then, the longitudinal vibration mode function of the beam is:

$\varphi_{u_{i}}(x)=D_{1_{i}} \cos \beta x_{i}+D_{2_{i}} \sin \beta x_{i}$

where $\beta^{2}=\rho \omega^{2} / E$.

\subsection{Bending vibration of LAB}

Consider the LAB as a Timoshenko beam, the dynamic equation of the bending vibration of LAB can be given as follows:

$\frac{\partial^{2} w_{1}}{\partial t^{2}}+\frac{E I_{1}}{\rho S_{1}} \frac{\partial^{4} w_{1}}{\partial x_{1}^{4}}-\frac{I_{1}}{S_{1}}\left(1+\frac{E}{\kappa G}\right) \frac{\partial^{4} w_{1}}{\partial x_{1}^{2} \partial t^{2}}+\frac{\rho I_{1}}{S_{1} \kappa G} \frac{\partial^{4} w_{1}}{\partial t^{2}}=0$,

where $w_{1}\left(x_{1}, t\right)$ is the transverse displacement of the beam, $\kappa$ is the section factor, $G$ is the shear modulus, $I_{1}$ is the second moment of the area of the LAB, and $S_{1}$ is the section area of the LAB. The effect of section moment of inertia is considered, while shear deformation is ignored. Thus, 
we obtain:

$\frac{\partial^{2} w_{1}}{\partial t^{2}}+\frac{E I_{1}}{\rho S_{1}} \frac{\partial^{4} w_{1}}{\partial x_{1}^{4}}-\frac{I_{1}}{S_{1}} \frac{\partial^{4} w_{1}}{\partial x_{1}^{2} \partial t^{2}}=0$.

Let $w_{1}\left(x_{1}, t\right)=\varphi_{w 1}\left(x_{1}\right) q(t)$. The bending vibration mode function of the LAB can then be shown as:

$\varphi_{w 1}(x)=C_{1} \cos s_{1} x_{1}+C_{2} \sin s_{1} x_{1}+C_{3} \cosh s_{2} x_{1}+C_{4} \sinh s_{2} x_{1}$

where:

$s_{1}=\sqrt{\sqrt{\gamma^{4}+\frac{\delta^{4}}{4}+\frac{\delta^{2}}{2}}}, \quad s_{2}=\sqrt{\sqrt{\gamma^{4}+\frac{\delta^{4}}{4}}-\frac{\delta^{2}}{2}}, \quad \gamma^{4}=\frac{\rho S_{1} \omega^{2}}{E I_{1}}, \quad \delta^{2}=\frac{\rho \omega^{2}}{E}$.

\subsection{Bending vibration of LFB}

The LFB is described as a Euler Bernoulli beam, and its axial deformation and transverse deformation in the $z$ direction are assumed to be negligible. The dynamic equations of the LFB is:

$\frac{\partial^{2}}{\partial x_{2}{ }^{2}}\left[E I_{2}\left(x_{2}\right) \frac{\partial^{2} w_{2}\left(x_{2}, t\right)}{\partial x_{2}^{2}}\right]+\rho S_{2}\left(x_{2}\right) \frac{\partial^{2} w_{2}\left(x_{2}, t\right)}{\partial t^{2}}=0$,

where $S_{2}\left(x_{2}\right)$ is the area of section, and $I_{2}(x)$ is the second moment of the area of the LFB. Let $w_{2}\left(x_{2}, t\right)=\varphi_{w 2}\left(x_{2}\right) q(t)$. The bending vibration mode function of the LFB is:

$\varphi_{w 2}(x)=B_{1} \cos p x_{2}+B_{2} \sin p x_{2}+B_{3} \cosh p x_{2}+B_{4} \sinh p x_{2}$,

where $p^{4}=\rho S^{2} \omega^{2} / E I^{2}$.

\subsection{Boundary conditions}

When are end of LFB (end A) is fixed, its boundary conditions are as follows:

$\left\{\begin{array}{l}\varphi_{w_{2}}\left(l_{2}\right)=0 \\ \varphi_{w_{2}}^{\prime}\left(l_{2}\right)=0 \\ \varphi_{u 2}\left(l_{2}\right)=0\end{array}\right.$

The distance between the hinge and the origin is approximately equal to the length of the LAB $\left(a \approx l_{1}\right.$ ), because mass $m$ is near the hinge. Thus, the boundary conditions of the end $\mathrm{D}$ of the $\mathrm{LAB}$ is:

$\left\{\begin{array}{l}\varphi_{w 1}\left(l_{1}\right)=0, \\ E I \varphi_{w 1}^{\prime \prime}\left(l_{1}\right)=-K \varphi_{w 1}^{\prime}\left(l_{1}\right) \\ \varphi_{u 1}\left(l_{1}\right)=0\end{array}\right.$

Considering the longitudinal and bending vibration at the junction of two beams, we obtain the displacement relationship between point $\mathrm{B}$ and $\mathrm{C}$ : 
$\left\{\begin{array}{l}u_{2} \sin \theta+w_{2} \cos \theta=w_{1}, \\ w_{2} \sin \theta-u_{2} \cos \theta=u_{1}, \\ \zeta_{B}=\zeta_{C},\end{array}\right.$

where $\zeta_{B}$ is the rotation angle of point $\mathrm{B}$, and $\zeta_{C}$ is the rotation angle of point $\mathrm{C}$. Based on continuity conditions, the equilibrium conditions of the generalized force of the point $\mathrm{B}$ and $\mathrm{C}$ can be obtained as:

$\left\{\begin{array}{l}M_{B}+M_{C}=0, \\ N_{B} \cos \theta+F_{B} \sin \theta+N_{C}=0, \\ N_{B} \sin \theta+F_{B} \cos \theta-F_{C}=0,\end{array}\right.$

where $M_{B(C)}$ is the bending moment of point $\mathrm{B}(\mathrm{C}), F_{B(C)}$ is the shearing force of point $\mathrm{B}(\mathrm{C})$, and $N_{B(C)}$ is the pulling force of point $\mathrm{B}(\mathrm{C})$. Substitute the boundary conditions Eq. (11) to Eq. (14), Eqs. (6), (8), and Eq. (10) yields:

$$
\left\{\begin{array}{l}
B_{1} \cos p l_{2}+B_{2} \sin p l_{2}+B_{3} \cosh p l_{2}+B_{4} \sinh p l_{2}=0, \\
-B_{1} \sin p l_{2}+B_{2} \cos p l_{2}+B_{3} \sinh p l_{2}+B_{4} \cosh p l_{2}=0, \\
D_{21} \cos \beta l_{2}+D_{22} \sin \beta l_{2}=0, \\
C_{1} \cos s_{1} a+C_{2} \sin s_{1} a+C_{3} \cosh s_{2} a+C_{4} \sinh s_{2} a=0, \\
\left(\begin{array}{l}
-C_{1} s_{1}^{2} \cos s_{1} a-C_{2} s_{1}^{2} \sin s_{1} a+C_{3} s_{2}^{2} \cosh s_{2} a+C_{4} s_{2}^{2} \sinh s_{2} a \\
=-\frac{K}{E I_{1}}\left(-C_{1} s_{1} \sin s_{1} a+C_{2} s_{1} \cos s_{1} a+C_{3} s_{2} \sinh s_{2} a+C_{4} s_{2} \cosh s_{2} a\right)
\end{array}\right), \\
D_{11} \cos \beta a+D_{12} \sin \beta a=0, \\
B_{1} \cos \theta+B_{3} \cos \theta-C_{1}-C_{3}+D_{21} \sin \theta=0, \\
B_{1} \sin \theta+B_{3} \sin \theta-D_{11}-D_{21} \cos \theta=0, \\
I_{2} p B_{2}+I_{2} p B_{4}-I_{1} s_{1} C_{2}-I_{1} s_{2} C_{4}=0, \\
-I_{2} p^{2} B_{1}+I_{2} p^{2} B_{3}-I_{1} s_{1}^{2} C_{1}+I_{1} s_{2}^{2} C_{3}=0, \\
-I_{2} p^{3} \sin \theta B_{2}+I_{2} p^{3} \sin \theta B_{4}+A_{1} \beta D_{12}+A_{2} \beta \cos \theta D_{22}=0, \\
-I_{2} p^{3} \cos \theta B_{2}+I_{2} p^{3} \cos \theta B_{4}+I_{1} s_{1}^{3} C_{2}-I_{1} s_{2}^{3} C_{4}+A_{2} \beta \sin \theta D_{22}=0,
\end{array}\right.
$$

where $l_{1}$ is the length of the LAB, $l_{2}$ is the length of the LFB, $\theta$ is the angle between the LFB and the horizontal direction. The coefficients $D_{1 i}(i=1,2), D_{2 i}, C_{j}(j=1,2,3,4), B_{j}$, and $\omega$ can be obtained through substitute of the boundary conditions.

\section{Results and discussions}

\subsection{Natural frequency and mode function}

The Eq. (15) is utilized in the free vibration analysis of the CMs to obtain the natural frequencies and vibration modes. The configuration parameters of the CMs are shown in Table 1. The material of the CMs is brass. Table 2 lists the first eight natural frequencies of the CMs. The vibration modes of the CMs are shown in Fig. 7. From Table 2 and Fig. 7, the following observations are worth noting.

1) In the first eight natural frequencies, the natural frequency of mode 3 and mode 4 is close, the difference is $333 \mathrm{~Hz}$; The natural frequency of mode 6 and mode 7 is close, the difference is $109 \mathrm{~Hz}$.

2) In the first five vibration modes, the longitudinal vibration of two beams (LAB and LFB) exist one peak. As the orders of the vibration modes increases, the position of the peak moves from the origin to the $x_{1}\left(x_{2}\right)$ direction. In mode 1 and mode 3 , the maximum deformation of the longitudinal vibration of the LAB is greater than that of the LFB. 

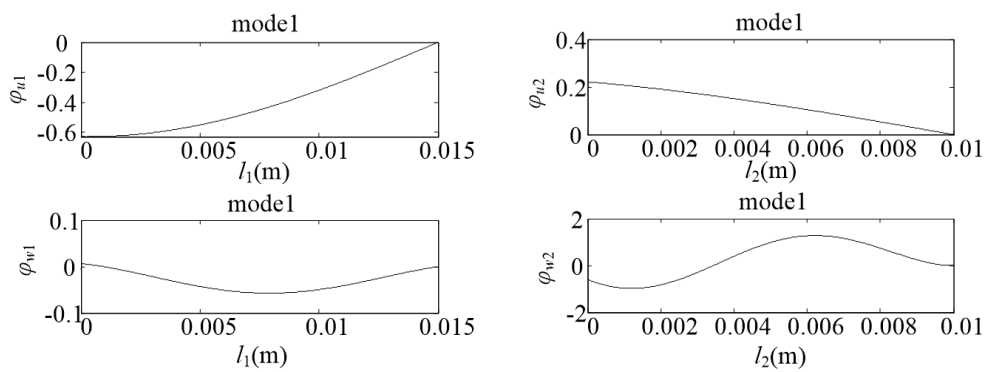

a)
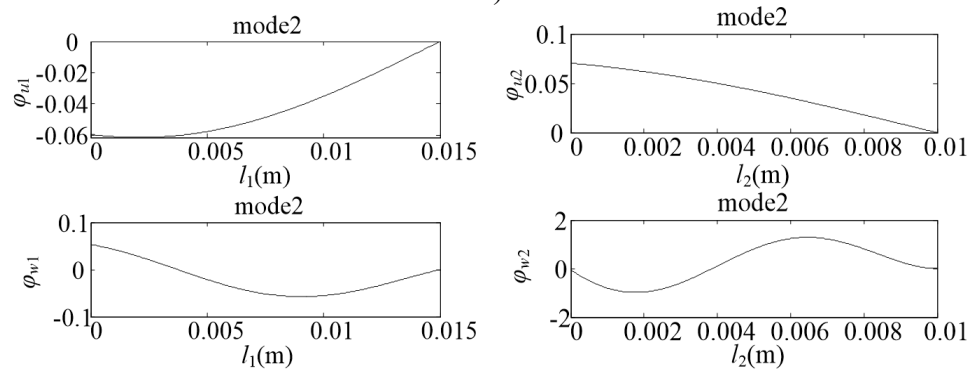

b)
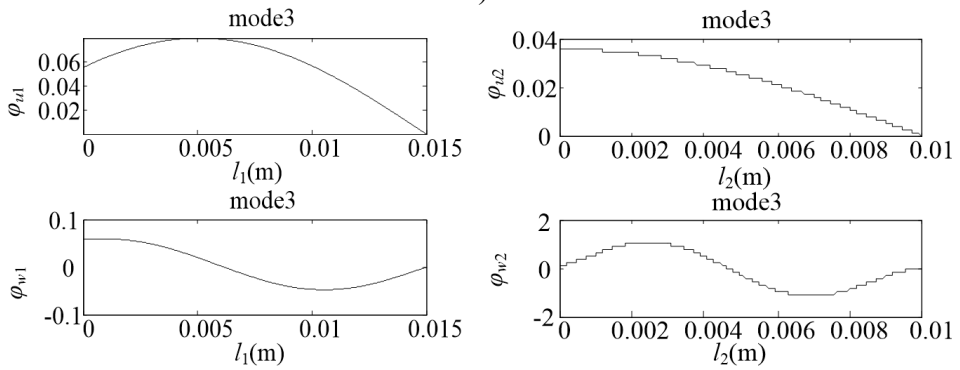

c)
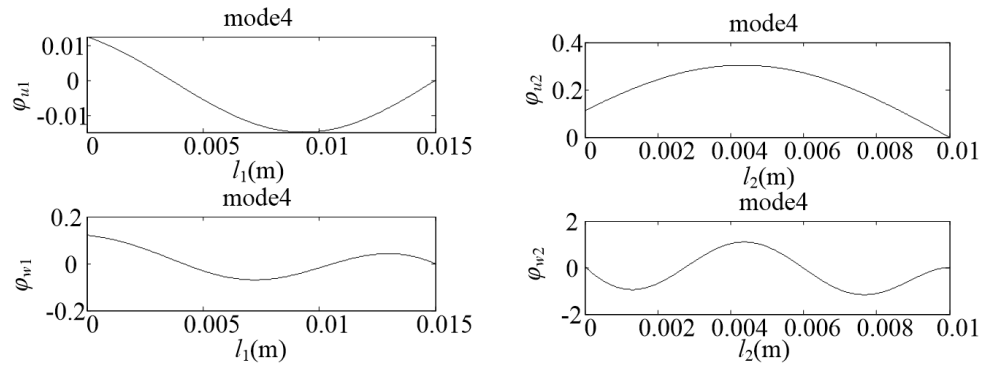

d)
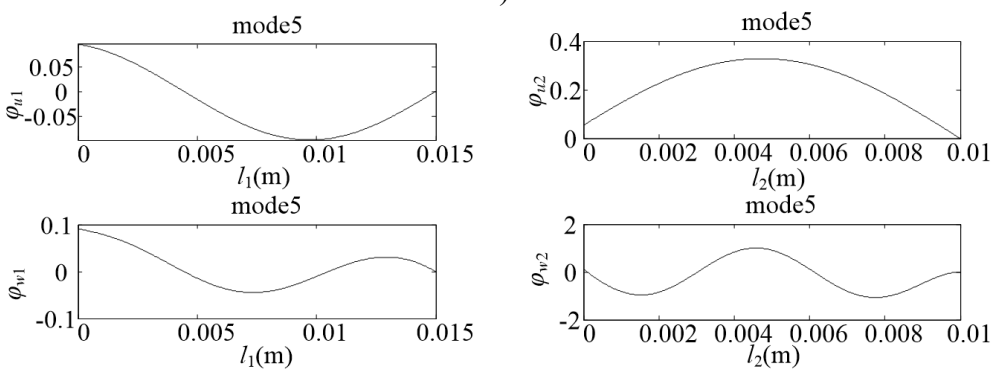

e)

Fig. 7. Vibration modes of the $\mathrm{CMs}$ 
3) The LAB has greater thickness than the LFB. Thus, the number of peaks of the LAB is less than that of the LFB in the same order of vibration mode. The maximum deformation of the bending vibration of the LAB is also less than that of the LFB.

Table 1. Parameters of the clamping mechanism

\begin{tabular}{|c|c|c|c|c|c|c|c|c|c|}
\hline$\rho\left(\mathrm{kg} / \mathrm{m}^{3}\right)$ & $E(\mathrm{GPa})$ & $b(\mathrm{~mm})$ & $t(\mathrm{~mm})$ & $h(\mathrm{~mm})$ & $l_{1}(\mathrm{~mm})$ & $h_{1}(\mathrm{~mm})$ & $l_{2}(\mathrm{~mm})$ & $h_{2}(\mathrm{~mm})$ & $\theta$ \\
\hline 8900 & 90 & 5 & 1 & 5 & 10 & 1 & 15 & 5 & $70^{\circ}$ \\
\hline
\end{tabular}

Table 2. Natural frequency $(\mathrm{Hz})$

\begin{tabular}{|c|c|c|c|c|c|c|c|}
\hline$\omega_{1}$ & $\omega_{2}$ & $\omega_{3}$ & $\omega_{4}$ & $\omega_{5}$ & $\omega_{6}$ & $\omega_{7}$ & $\omega_{8}$ \\
\hline 1704 & 1948 & 2535 & 4435 & 4768 & 5431 & 5540 & 7900 \\
\hline
\end{tabular}

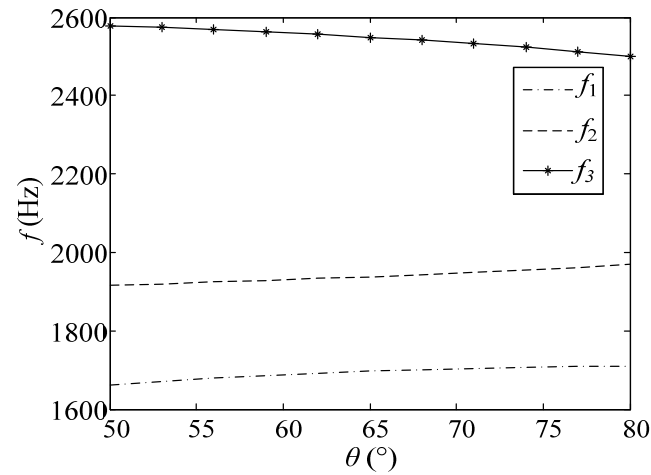

a) $\theta$ change

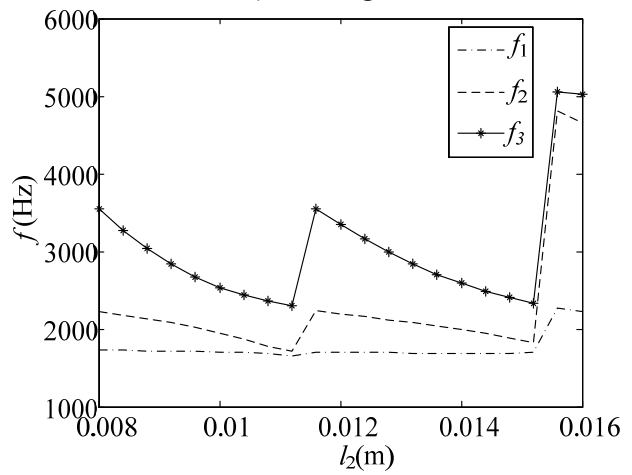

c) $l_{2}$ change

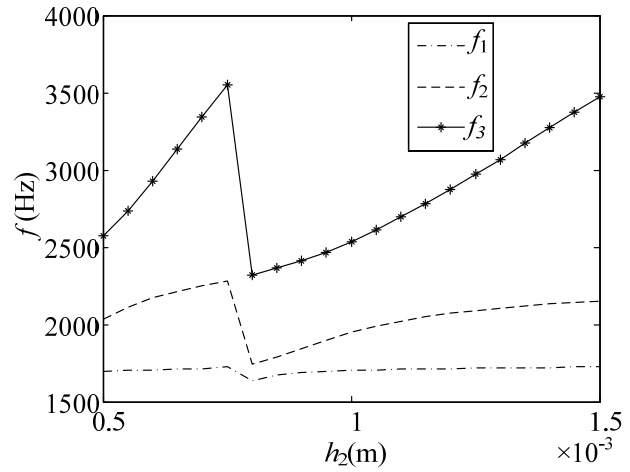

e) $h_{2}$ change

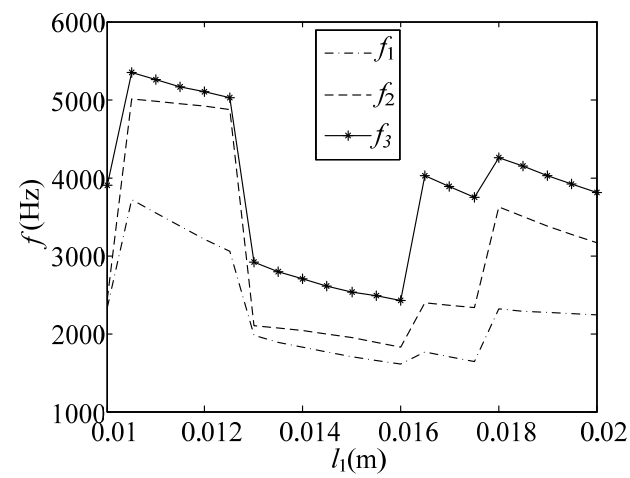

b) $l_{1}$ change

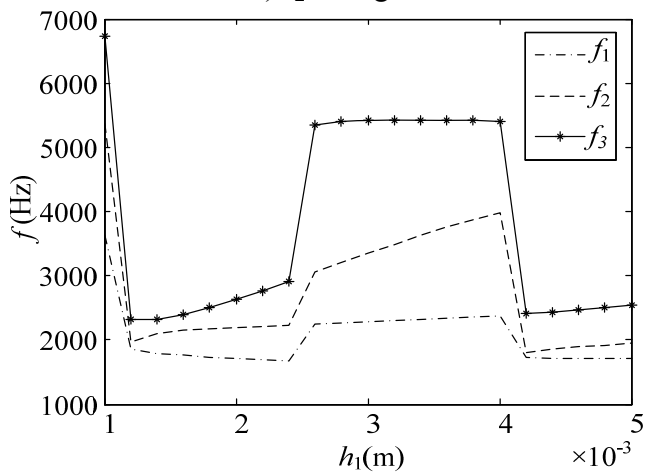

d) $h_{1}$ change

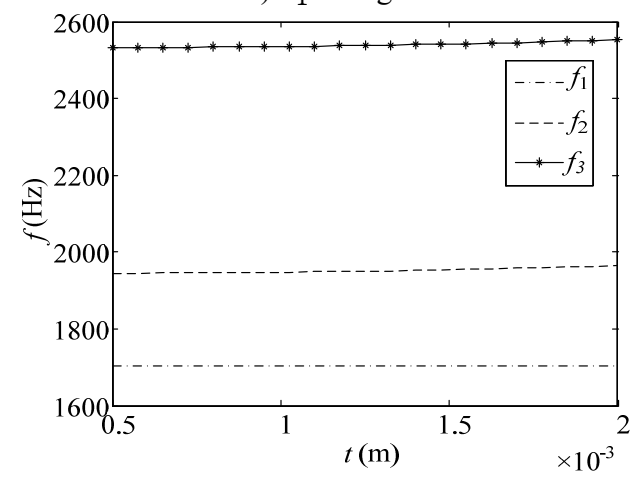

f) $t$ change

Fig. 8. Changes of natural frequencies with respect to various parameters 


\subsection{Sensibility analysis}

We investigate the changes of CMs natural frequency along with the configuration parameters. The changes of natural frequency are shown in Fig. 8. The materials of the CMs and corresponding first three natural frequencies are shown in Table 3.

Table 3. The first three natural frequency of different material

\begin{tabular}{|l|c|c|c|c|c|}
\hline \multirow{2}{*}{ Material } & Modulus of elasticity & \multirow{2}{*}{\begin{tabular}{c} 
Density \\
\cline { 4 - 6 }
\end{tabular}} & $E\left(\mathrm{~N} / \mathrm{m}^{2}\right)$ & \multicolumn{3}{|c|}{ Frequency } \\
\cline { 4 - 6 } & $65 \times 10^{9}$ & $2.7 \times 10^{3}$ & 2629 & 3005 & 3912 \\
\hline Aluminum & $206 \times 10^{9}$ & $7.7 \times 10^{3}$ & 2771 & 3168 & 4124 \\
\hline Carbon steel & $90 \times 10^{9}$ & $8.9 \times 10^{3}$ & 1704 & 1947 & 2535 \\
\hline Brass & $206 \times 10^{9}$ & $7.9 \times 10^{3}$ & 2736 & 3127 & 4071 \\
\hline Alloy steel & $120 \times 10^{9}$ & $7 \times 10^{3}$ & 2218 & 2535 & 3301 \\
\hline Gray pig iron & & \multicolumn{4}{|c|}{} \\
\hline
\end{tabular}

The following observations are drawn:

1) As the horizontal angle of the LFB changes between $50^{\circ}$ and $80^{\circ}$, the first-order and second-order natural frequency grows nonlinear, while the third natural frequency drops nonlinear.

2) When the length and thickness of the LAB and LFB change, the change in the natural frequency is irregularly serrate. In the change interval for some parameters $\left(l_{1}, h_{1}, l_{2}, h_{2}\right)$, the natural frequency drops as the length of the LAB and LFB increases, the natural frequency drops; With the increase of the thickness of the LAB and the LFB, the natural frequency nonlinearity increases. When the parameters $\left(l_{1}, h_{1}, l_{2}, h_{2}\right)$ change to a certain value, (e.g., $l_{1}=10.5,12.5$, $\left.16.5,18 \mathrm{~mm}, l_{2}=11.6,15.6 \mathrm{~mm}, h_{1}=1.2,2.6,4, h_{2}=0.8 \mathrm{~mm}\right)$, the curve of the natural frequency changes and mutates. This phenomenon can be attributed to the mutation of the vibration modes of the CMs under these parameters values.

3) When the notch thickness of the flexible hinge changes between 0.5 and $2 \mathrm{~mm}$, the natural frequency of the CMs slowly increases. The growth trend of the natural frequency becomes significant as the order number increases.

4) The natural frequency of each order is lower when brass is used as CMs material than when other materials are used. By contrast, the natural frequency of each order is higher when carbon steel is used as CMs material than when other materials are used.

\section{TEST}

The natural frequencies of the CMs are obtained by using the SZCJ knocking vibration tests system (see Fig. 9). The experimental results are shown in Fig. 10.

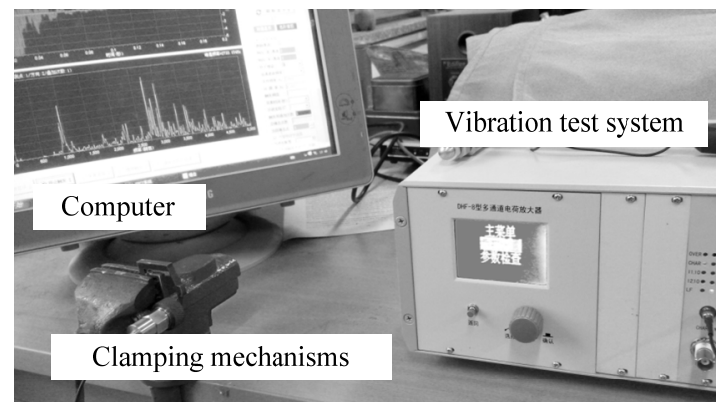

Fig. 9. Impact hammer modal test

The relative errors are obtained by comparing the theoretical values and the experimental results. Table 4 shows that the relative errors in the first three natural frequencies are $2.7 \%, 2.7 \%$, 
and $2.6 \%$. These relative errors are less than $3.0 \%$ suggesting the reasonable agreement of the experimental results with the results of our theoretical analysis.

Table 4. Comparison of experimental results and theoretical values

\begin{tabular}{|l|c|c|c|}
\hline \multicolumn{1}{|c|}{ Frequency } & $\omega_{1}(\mathrm{~Hz})$ & $\omega_{2}(\mathrm{~Hz})$ & $\omega_{3}(\mathrm{~Hz})$ \\
\hline Theoretical values & 1704 & 1948 & 2535 \\
\hline Experimental results & 1751.7 & 2002.5 & 2602.5 \\
\hline Relative errors & $2.7 \%$ & $2.7 \%$ & $2.6 \%$ \\
\hline
\end{tabular}

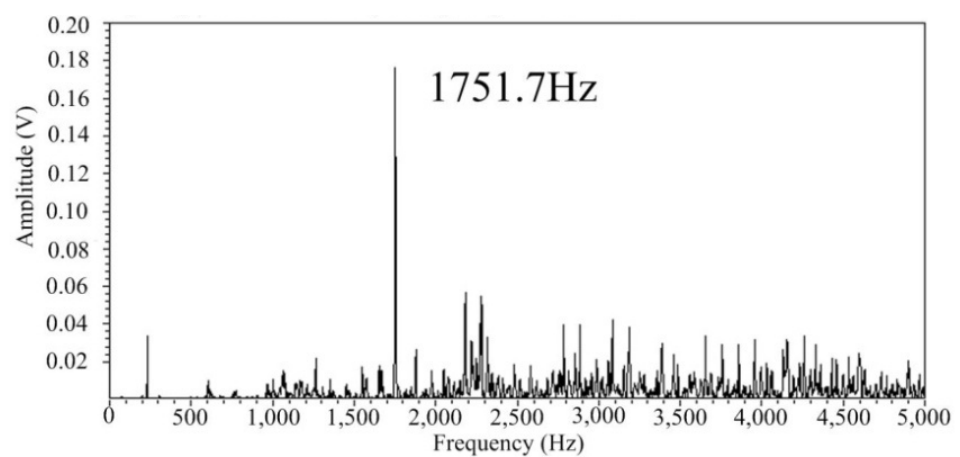

a) Mode 1

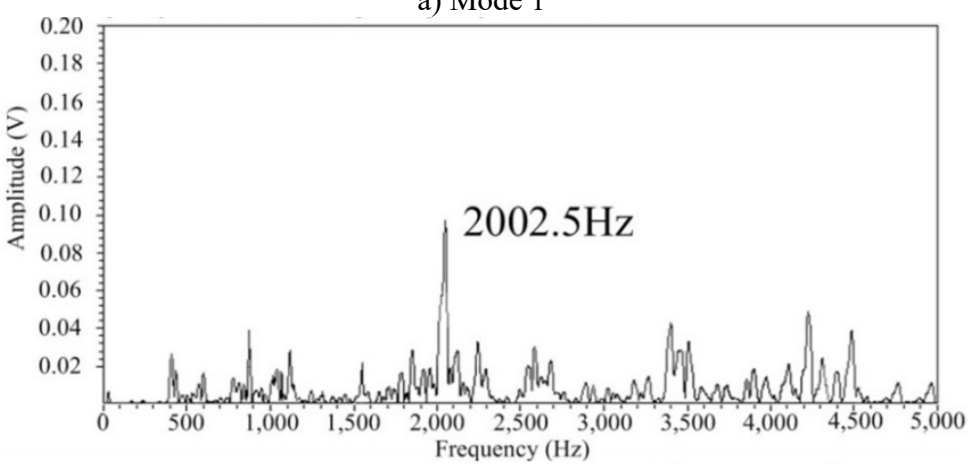

b) Mode 2

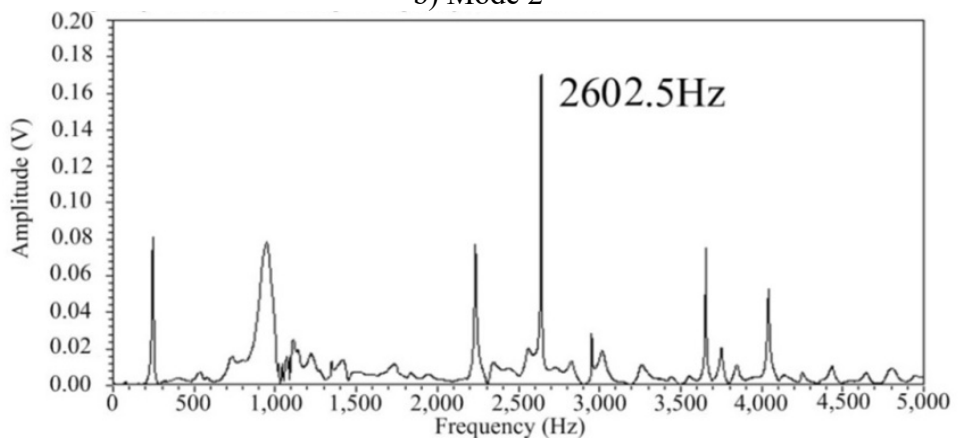

c) Mode 3

Fig. 10. Experimental results (Sample frequency $=10000.00 \mathrm{~Hz})$

\section{Conclusions}

A novel rotary inchworm piezoelectric motor was proposed and the drive principle was discussed. The dynamic equations of the CMs were established. The natural frequencies and vibration modes were obtained, and the sensibility of the CMs was investigated. The relative errors of the natural frequency between the theoretical and experimental results verify the correctness of 
the theoretical analysis. This dynamic analysis of the CMs lays a theoretical foundation for the design of a novel rotary inchworm piezoelectric motor.

\section{Acknowledgement}

This project is supported by the Independent Study Program for Young Teachers of Yanshan University (13LGB002).

\section{References}

[1] Duong Khanh, Garcia Ephrahim Design and performance of a rotary motor driven by piezoelectric stack actuators. Japanese Journal of Applied Physics, Vol. 35, Issue 12, 1996, p. 6334-6341.

[2] Vaughan Mark, Leo Donald J. Integrated piezoelectric linear motor for vehicle applications. ASME International Adaptive Structures and Materials Systems Symposium, New Orleans, 2002, p. 1-9.

[3] Cusin P., Sawai T., Konishi S. Compact and precise positioner based on the inchworm principle. Journal of Micromechanics and Microengineering, Vol. 10, Issue 4, 2000, p. 516-521.

[4] Duong Khanh, Garcia Ephrahim Development of a rotary inchworm piezoelectric motor. Smart Structures and Materials: Smart Structures and Integrated Systems, Vol. 2443, 1995, p. 752-788.

[5] Morita T., Murakami H., Yokose T., Hosaka H. A miniaturized resonant-type smooth impact drives mechanism actuator. Sensors and Actuators A, Vol. 178, 2012, p. 188-192.

[6] Tenzer P. E., Ben M. R. A systematic procedure for the design of piezoelectric inchworm precision position. IEEE/ASME Transactions on Mechatronics, Vol. 9, Issue 2, 2004, p. 427-435.

[7] Li J., Ramin S., Javad D. Design and development of a new piezoelectric linear inchworm actuator. Mechatronics, Vol. 15, 2005, p. 651-681.

[8] Wu Yingfei, Zhou Zhaoying Design calculations for flexure hinge. Review of Scientific Instruments, Vol. 73, 2002, p. 3101-3106.

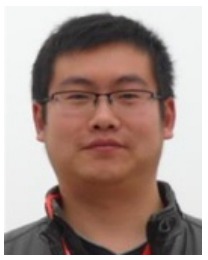

Yongfei Gu received M.S. degree in School of Mechanical Engineering from Yanshan University, Qinhuangdao, China, in 2008. Now he works at Yanshan University. His current research interests include piezoelectric driving and control, advanced forging and stamping technology.

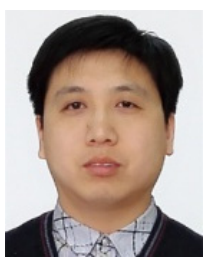

Jichun Xing received Ph.D. degree in School of Mechanical Engineering from Yanshan University, Qinhuangdao, China, in 2012. Now he works at Yanshan University. His current research interests include piezoelectric driving and control and haptics technology. 